\title{
Iterations of the Frobenius-Perron operator for parabolic random maps
}

by

\author{
Zbigniew S. Kowalski (Wrocław)
}

\begin{abstract}
We describe totally dissipative parabolic extensions of the one-sided Bernoulli shift. For the fractional linear case we obtain conservative and totally dissipative families of extensions. Here, the property of conservativity seems to be extremely unstable.
\end{abstract}

0. Introduction. Let $\sigma$ be the one-sided $(p, q)$-Bernoulli shift on the space $\Omega=\{0,1\}^{\mathbb{N}}, \mathbb{N}=\{0,1,2, \ldots\}$, with the $(p, q)$-measure $\mu_{p}$ on $(\Omega, \mathcal{B})$, where $\mathcal{B}$ is the Borel product $\sigma$-algebra and $(p, q)$ is a probability vector. Let us consider two transformations $T_{0}, T_{1}$ of the interval $[0,1]$ onto itself such that $T_{i} \in C^{2}[0,1], T_{i}^{\prime}>0, T_{i}(0)=0, T_{i}(1)=1$ for $i=0,1$ and $T_{0} \geq I$, $T_{1} \leq I$ where $I(x)=x$ for $x \in[0,1]$. Let $S_{i}$ denote the inverse of $T_{i}, i=0,1$. We define the transformation

$$
T(\omega, x)=\left(\sigma(\omega), S_{\omega(0)}(x)\right) .
$$

This transformation is a realization of the random map $T(x)=S_{0}(x)$ with probability $p$ and $T(x)=S_{1}(x)$ with probability $q$, or a realization of the random walk on the unit interval. Let $\Lambda$ denote the Lebesgue measure on $[0,1]$. It will cause no confusion to use the same letter for the Lebesgue measure on $\mathbb{R}$. Moreover, let us denote by $P$ the restriction to $L^{1}(\Lambda)$ of the Frobenius-Perron operator with respect to $\mu_{p} \times \Lambda$. By using two different methods we investigate iterations of $P$. The first has been used for transformations $T$ such that

$$
T_{i}=\left(1-\varepsilon_{i}\right) x+\varepsilon_{i} g(x), \quad i=0,1,
$$

$g \in C^{2}[0,1], g(0)=0, g(1)=1,\left(1-\sup g^{\prime}\right)^{-1}<\varepsilon_{0}, \varepsilon_{1}<\left(1-\inf g^{\prime}\right)^{-1}$. We additionally assume that there exists exactly one point $x_{0}$ for which

2000 Mathematics Subject Classification: Primary 37A40.

Key words and phrases: Bernoulli shift, parabolic extension, conservative, totally dissipative, Frobenius-Perron operator. 
$g^{\prime}\left(x_{0}\right)=1$ and $g^{\prime}(x)<1$ for $x<x_{0}$ or $g^{\prime}(x)>1$ for $x<x_{0}$. By the modification of $P$ to $P_{h}$ which relies on replacing $\Lambda$ by the equivalent measure with density $h$ we show that $P_{h}^{n}(1) \rightarrow 0$, which yields the total dissipativity of $T$ for some parameters $\varepsilon_{i}, i=0,1$, and $p$ for $g(x)=x^{2}$. We also observe that $\left(T, \mu_{p} \times \Lambda\right)$ for $T$ given by (2) is either conservative and ergodic, or totally dissipative. The conservativity of different kinds of random maps is studied in [D-K-S]. In the second method we apply the isomorphism of fractional linear maps with translations of the real line $\mathbb{R}$. By using the results about conservativity of $\mathbb{R}$-extensions, we obtain either conservative and ergodic, or totally dissipative systems which have an equivalent $\sigma$-finite invariant measure. The conservative transformations appear to be isolated. Moreover, by repeating the approximation argument from [K3] we extend the area of dissipativity of $T$ given by (2) for $g(x)=x^{2}$. The observation that fractional linear extensions are isomorphic to random walks on $\mathbb{R}$ allows us to improve the description of their ergodic properties included in [K3]. We finish our paper by completing the information about the example of [K2], i.e. the transformation $T$ given by $T_{0}=\frac{3}{2} x-\frac{1}{2} x^{2}, T_{1}=x^{2}$ and $p \in(0,1)$.

1. Total dissipativity. We start with a slightly more general situation, i.e. $\sigma$ is the one-sided Markov shift on the space $\Omega=\{0, \ldots, s-1\}^{N}, s \geq$ 2 , with $(\Pi, \vec{p})$-measure $\mu_{\vec{p}}$. Here $\vec{p}=\left(p_{0}, \ldots, p_{s-1}\right)$ is a probability vector, and $\Pi=\left(p_{i j}\right)_{s \times s}$ is a stochastic matrix such that $\vec{p} \Pi=\vec{p}$. Let $\left\{S_{i}\right\}_{i=0}^{s-1}$ be a family of positively and negatively nonsingular transformations of a probability space $(Y, \mathcal{C}, m)$, i.e. $m(B)=0 \Rightarrow m\left(S_{i}^{-1}(B)\right)=m\left(S_{i}(B)\right)=0$ for $i=0, \ldots, s-1$. This definition slightly differs from that of the two-sided nonsingularity $[\mathrm{A}]$. We introduce the transformation

$$
T(\omega, x)=\left(\sigma(\omega), S_{\omega(0)}(x)\right) .
$$

Let us denote by $\mathcal{C}(T)$ the conservative part of $T$ and by $\mathcal{D}(T)$ the dissipative part. Moreover, let $\alpha=\left\{A_{i}: i=0, \ldots, s-1\right\}$ where $A_{i}=\{\omega: \omega(0)=i\}$.

Theorem 1. If $E \subset \mathcal{C}(T), \mu_{\vec{p}} \times m(E)>0$ and $T(E) \subset E$ then

$$
E=\bigcup_{i=0}^{s-1} A_{i} \times E_{i} \quad \text { for some } E_{i} \in \mathcal{C} .
$$

Proof. Let $E_{x}=\{\omega:(\omega, x) \in E\}$. Suppose, on the contrary, that there exist $\epsilon>0$ and $i$ such that

$$
B=\left\{x: 0<\mu_{\vec{p}}\left(E_{x} \cap A_{i}\right) \leq(1-\epsilon) \mu_{\vec{p}}\left(A_{i}\right)\right\}
$$

has positive measure $m$. Let $(\omega, x) \in E \cap A_{i} \times B$, where $\omega$ is a density point for $E_{x}$ and $T^{n}(\omega, x)$ returns infinitely many times to $E \cap A_{i} \times B$ (because 
$E \subset \mathcal{C}(T))$. By the choice of $\omega$, there exists $n_{0}$ such that for $n \geq n_{0}$,

$$
\mu_{\vec{p}}\left(A_{n}(\omega) \cap E_{x}\right) \geq(1-\epsilon / 2) \mu_{\vec{p}}\left(A_{n}(\omega)\right)
$$

where $\omega \in A_{n}(\omega) \in \bigvee_{i=0}^{n} \sigma^{-i} \alpha$. Let $n_{1}$ satisfy $n_{1}>n_{0}$ and $T^{n_{1}}(\omega, x) \in$ $E \cap A_{i} \times B$. Then

$$
T^{n_{1}}\left(A_{n_{1}}(\omega) \cap E_{x} \times\{x\}\right)=\sigma^{n_{1}}\left(A_{n_{1}}(\omega) \cap E_{x}\right) \times\left\{S_{\omega}^{n_{1}}(x)\right\}
$$

where $S_{\omega}^{n_{1}}(x)=S_{\omega\left(n_{1}-1\right)} \circ \cdots \circ S_{\omega(0)}(x)$. Hence

$$
E_{S_{\omega}^{n_{1}}(x)} \cap A_{i} \supset \sigma^{n_{1}}\left(A_{n_{1}}(\omega) \cap E_{x}\right) .
$$

Therefore

$$
\begin{aligned}
\mu_{\vec{p}}\left(E_{S_{\omega}^{n_{1}}(x)} \cap A_{i}\right) & \geq J_{\sigma^{n_{1}}}(\omega) \mu_{\vec{p}}\left(A_{n_{1}}(\omega) \cap E_{x}\right) \\
& \geq(1-\epsilon / 2) J_{\sigma^{n_{1}}}(\omega) \mu_{\vec{p}}\left(A_{n_{1}}(\omega)\right)=(1-\epsilon / 2) \mu_{\vec{p}}\left(A_{i}\right) .
\end{aligned}
$$

Here $J_{\sigma}$ denotes the Jacobian of $\sigma$. This contradicts our assumption.

Corollary 1. $\mathcal{C}(T)=\bigcup_{i=0}^{s-1} A_{i} \times B_{i}$ and $\mathcal{D}(T)=\bigcup_{i=0}^{s-1} A_{i} \times C_{i}$.

TheOREM 2. If $T$ is given by (2) then $\left(T, \mu_{p} \times \Lambda\right)$ is either conservative and ergodic, or totally dissipative.

Proof. Let $\mu_{p} \times \Lambda(\mathcal{C}(T))>0$. Hence by Corollary $1, \mathcal{C}(T)=\Omega \times B$. Moreover, $T(\Omega \times B)=\Omega \times B$, which implies $\Lambda\left(B \div\left(S_{0} B \cup S_{1} B\right)\right)=0$. Therefore, $T(\Omega \times B) \subset \Omega \times B$ with respect to the measure $\mu_{r} \times \Lambda$ where $r \epsilon_{0}+(1-r) \epsilon_{1}=0$. The measure $\mu_{r} \times \Lambda$ is $T$-invariant and ergodic (for the proof see [K1]). Thus, $\Lambda(B)=1$. We apply similar arguments to get the ergodicity of $T$.

Let $h:(0,1) \rightarrow \mathbb{R}^{+}$be a function from $C^{1}(0,1)$ and $\nu_{h}$ be a measure on $[0,1]$ such that $d \nu_{h} / d \Lambda=h$. For our applications we use $h(x)=x^{-\alpha}$ or $h(x)=(1-x)^{-\alpha}$ for $\alpha \geq 1$. Let $T$ be the transformation given by (1) and let $\mathcal{P}\left(\mathcal{P}_{h}\right.$ respectively) be the restriction to $L^{1}(\Lambda)\left(L^{1}\left(\nu_{h}\right)\right.$ respectively) of the Frobenius-Perron operator with respect to $\mu_{p} \times \Lambda$ ( $\mu_{p} \times \nu_{h}$ respectively). The following relation holds between these operators:

We define

$$
\mathcal{P}_{h} f=h^{-1} \mathcal{P}(h f) \quad \text { for } f \in L^{1}\left(\nu_{h}\right) .
$$

$$
\begin{aligned}
& h^{-1} h\left(T_{i}\right)(0)=\lim _{x \rightarrow 0^{+}} h^{-1}(x) h\left(T_{i}(x)\right), \\
& h^{-1} h\left(T_{i}\right)(1)=\lim _{x \rightarrow 1^{-}} h^{-1}(x) h\left(T_{i}(x)\right) \quad \text { for } i=0,1 .
\end{aligned}
$$

Here we assume the existence of the above limits. The explicit form of $\mathcal{P}_{h}$ is

$$
\begin{aligned}
\mathcal{P}_{h} f(x)= & p h\left(T_{0}(x)\right) h^{-1}(x) T_{0}^{\prime}(x) f\left(T_{0}(x)\right) \\
& +(1-p) h\left(T_{1}(x)\right) h^{-1}(x) T_{1}^{\prime}(x) f\left(T_{1}(x)\right),
\end{aligned}
$$

for $x \in[0,1]$. Our aim is to obtain some conditions for dissipativity of $T$. 
Theorem 3. Let $T$ be given by (1). If for all $x \in[0,1]$, $\gamma(x)=p h^{-1}(x) h\left(T_{0}(x)\right) T_{0}^{\prime}(x)+(1-p) h^{-1}(x) h\left(T_{1}(x)\right) T_{1}^{\prime}(x) \leq \beta<1$ then $T$ is totally dissipative.

Proof. By assumption, $P_{h}(1) \leq \beta$. Therefore $P_{h}^{n}(1) \leq \beta^{n}$. Let $f \in L^{1}(\Lambda)$ be such that $0<f \leq h$. Then

$$
P^{n}(f)=P^{n}\left(h \frac{f}{h}\right)=h P_{h}^{n}\left(\frac{f}{h}\right) \leq h P_{h}^{n}(1) \leq \beta^{n} h .
$$

Therefore, the measure of $\left\{x: \sum_{n=0}^{\infty} P^{n} f<\infty\right\}$ is equal to one. This proves the theorem.

2. An application. Let us consider the transformations

$$
T_{0}(x)=\left(1+\epsilon_{0}\right) x-\epsilon_{0} x^{2}, \quad T_{1}(x)=\left(1-\epsilon_{1}\right) x+\epsilon_{1} x^{2},
$$

for $\epsilon_{0}, \epsilon_{1} \in[0,1]$. For $h(x)=x^{-2}$ we determine $p$ which satisfies

$$
\gamma(x)=p \frac{1+\epsilon_{0}-2 \epsilon_{0} x}{\left(1+\epsilon_{0}-\epsilon_{0} x\right)^{2}}+(1-p) \frac{1-\epsilon_{1}+2 \epsilon_{1} x}{\left(1-\epsilon_{1}+\epsilon_{1} x\right)^{2}}<1
$$

for every $x \in[0,1]$. For this purpose we compute

$$
\gamma^{\prime}(x)=-2 p \epsilon_{0}^{2} \frac{x}{\left(1+\epsilon_{0}-\epsilon_{0} x\right)^{3}}-2(1-p) \epsilon_{1}^{2} \frac{x}{\left(1-\epsilon_{1}+\epsilon_{1} x\right)^{3}} \leq 0 .
$$

Therefore, $\gamma(x) \leq \gamma(0)$ for $x \in[0,1]$. Hence for $\epsilon_{1} \neq 1$ we get

$$
\beta=\gamma(0)<1 \Leftrightarrow \frac{p}{1+\epsilon_{0}}+\frac{1-p}{1-\epsilon_{1}}<1 \Leftrightarrow p>\frac{1+\epsilon_{0}}{\epsilon_{1}+\epsilon_{0}} \epsilon_{1} .
$$

The same reasoning applies to the case $h(x)=(1-x)^{-2}$. For $\epsilon_{0} \neq 1$ we get

$$
\beta=\gamma(1)<1 \Leftrightarrow \frac{p}{1-\epsilon_{0}}+\frac{1-p}{1+\epsilon_{1}}<1 \Leftrightarrow p<\frac{1-\epsilon_{0}}{\epsilon_{1}+\epsilon_{0}} \epsilon_{1} .
$$

Therefore as a consequence of Theorem 3 we get

Corollary 2. If $T$ is given by (3) then $\left(T, \mu_{p} \times \Lambda\right)$ is totally dissipative whenever

$$
p<\frac{1-\epsilon_{0}}{\epsilon_{1}+\epsilon_{0}} \epsilon_{1} \quad \text { or } \quad p>\frac{1+\epsilon_{0}}{\epsilon_{1}+\epsilon_{0}} \epsilon_{1} .
$$

We can improve on the above by using $h(x)=x^{-(1+\alpha)}$ or $h(x)=$ $(1-x)^{-(1+\alpha)}$ for $\alpha \in(0,1)$.

EXAMPLE. For

$$
h(x)=x^{-1.4}, \quad p \geq 0.77, \quad \epsilon_{0}=0.9, \quad \epsilon_{1}=0.7
$$

we get $\gamma(x)<1$ for every $x \in[0,1]$. Similarly for

$$
h(x)=(1-x)^{-1.4}, \quad p \leq 0.4, \quad \epsilon_{0}=0.5, \quad \epsilon_{1}=1
$$

we get $\gamma(x)<1$ for each $x \in[0,1]$. 
3. Fractional linear maps and $\mathbb{R}$-extensions. Let $T$ be given by (1) where

$$
\begin{array}{ll}
T_{0}=T_{\lambda_{0}}=\frac{x}{\lambda_{0} x+1-\lambda_{0}}, & \lambda_{0} \in(0,1), \\
T_{1}=T_{\lambda_{1}}=\frac{x}{\lambda_{1} x+1-\lambda_{1}}, & \lambda_{1}<0 .
\end{array}
$$

REMARK 1 . $T$ has an equivalent invariant $\sigma$-finite measure for every $p \in(0,1)$.

Proof. It is easy to see that the measure $\mu_{p} \times \nu$ where $d \nu / d \Lambda=1 / x(1-x)$ is $T$-invariant.

Let us observe that the system $\left(\Omega \times[0,1], \mu_{p} \times \nu, T\right)$, where $T$ and $\nu$ are considered above, is isomorphic to $\left(\Omega \times \mathbb{R}, \mu_{p} \times \Lambda, \hat{T}\right)$ where

$$
\hat{T}(\omega, u)=\left(\sigma(\omega), u+a_{\omega(0)}\right) .
$$

Here $a_{0}=\ln \left(1-\lambda_{0}\right)$ and $a_{1}=\ln \left(1-\lambda_{1}\right)$. The isomorphism is given by the map

$$
\Omega \times \mathbb{R} \ni(\omega, u) \mapsto\left(\omega, \frac{e^{u}}{1+e^{u}}\right) \in \Omega \times[0,1] .
$$

Now we are in a position to use Corollary 8.15 of [A].

THEOREM 4. $T$ is conservative if and only if

$$
p=\frac{\ln \left(1-\lambda_{1}\right)}{\ln \left(\frac{1-\lambda_{1}}{1-\lambda_{0}}\right)} .
$$

For other $p, T$ is totally dissipative.

The second observation relies on the representation of $\hat{T}$ as a random walk on $\mathbb{R}$. Namely, $\left(\Omega \times \mathbb{R}, \mu_{p} \times \Lambda, \hat{T}\right)$ is isomorphic to $\left(\mathbb{R}^{N}, \mu, \sigma\right)$ via the map

$$
\Phi(\omega, u)=\left(u, u+a_{\omega(0)}, u+a_{\omega(0)}+a_{\omega(1)}, \ldots\right) \in \mathbb{R}^{N} .
$$

Here $\sigma$ is the one-sided shift and $\mu$ is determined by the "jump probability"

$$
P=p \delta_{\left\{\ln \left(1-\lambda_{0}\right)\right\}}+(1-p) \delta_{\left\{\ln \left(1-\lambda_{1}\right)\right\}}
$$

and $\Lambda$.

TheOREM 5. $\left(\Omega \times[0,1], \mu_{p} \times \nu, T\right)$ is

(i) ergodic if and only if $\frac{\ln \left(1-\lambda_{1}\right)}{\ln \left(1-\lambda_{0}\right)}$ is irrational,

(ii) not exact.

Proof. By results of $[\mathrm{D}-\mathrm{L}]$ the random walk $\left(\mathbb{R}^{N}, \mu, \sigma\right)$ is ergodic if and only if the set

$$
\left\{n \ln \left(1-\lambda_{0}\right)+m \ln \left(1-\lambda_{1}\right): m, n \in \mathbb{Z}\right\}
$$


is dense in $\mathbb{R}$. But the above is equivalent to

$$
\frac{\ln \left(1-\lambda_{1}\right)}{\ln \left(1-\lambda_{0}\right)} \notin \mathbb{Q}
$$

Moreover, $\left(\mathbb{R}^{N}, \mu, \sigma\right)$ is exact if and only if

$$
\left\{n \ln \left(\frac{1-\lambda_{1}}{1-\lambda_{0}}\right): n \in \mathbb{Z}\right\}
$$

is dense in $\mathbb{R}$. But this is impossible.

The isomorphism of $T$ and $\hat{T}$ carries new information about iterations of $T$. Namely,

$$
\hat{T}^{n}(\omega, u)=\left(\sigma^{n}(\omega), u+\left(n-S_{n}(\omega)\right) a_{0}+S_{n}(\omega) a_{1}\right)
$$

where

$$
S_{n}(\omega)=\sum_{k=0}^{n-1} \omega(k) .
$$

Therefore,

$$
u+\left(n-S_{n}(\omega)\right) a_{0}+S_{n}(\omega) a_{1} \rightarrow \infty
$$

and simultaneously

$$
1_{\Omega \times[0, b]}\left(T^{n}(\omega, x)\right) \rightarrow 0 \quad \text { for a.e. } \omega
$$

when

$$
p<\frac{\ln \left(1-\lambda_{1}\right)}{\ln \left(\frac{1-\lambda_{1}}{1-\lambda_{0}}\right)} .
$$

Moreover,

$$
u+\left(n-S_{n}(\omega)\right) a_{0}+S_{n}(\omega) a_{1} \rightarrow-\infty
$$

and at the same time

$$
1_{\Omega \times[b, 1]}\left(T^{n}(\omega, x)\right) \rightarrow 0 \quad \text { for a.e. } \omega
$$

if

$$
\frac{\ln \left(1-\lambda_{1}\right)}{\ln \left(\frac{1-\lambda_{1}}{1-\lambda_{0}}\right)}<p
$$

Here $b \in(0,1)$. We will apply the last observations to parabolic extensions $T$ given by (3). It is easy to see that

$$
\operatorname{sgn}\left(T_{0}(x)-T_{\lambda_{0}}(x)\right)=\operatorname{sgn}\left(\epsilon_{0} \lambda_{0} x-\epsilon_{0} \lambda_{0}+\epsilon_{0}-\lambda_{0}\right)
$$

and

$$
\operatorname{sgn}\left(T_{1}(x)-T_{\lambda_{1}}(x)\right)=\operatorname{sgn}\left(-\epsilon_{1} \lambda_{1} x+\epsilon_{1} \lambda_{1}-\epsilon_{1}-\lambda_{1}\right)
$$

for $x \in(0,1)$. Therefore, we get

$$
S_{0}(x) \geq T_{\lambda_{0}}^{-1}(x) \Leftrightarrow \epsilon_{0} \leq \lambda_{0}, \quad S_{1}(x) \geq T_{\lambda_{1}}^{-1}(x) \Leftrightarrow-\epsilon_{1} \leq \lambda_{1}
$$


and

$$
\begin{aligned}
& S_{0}(x) \leq T_{\lambda_{0}}^{-1}(x) \Leftrightarrow \lambda_{0} \leq \frac{\epsilon_{0}}{1+\epsilon_{0}}, \\
& S_{1}(x) \leq T_{\lambda_{1}}^{-1}(x) \Leftrightarrow \lambda_{1} \leq-\frac{\epsilon_{1}}{1-\epsilon_{1}}, \quad \epsilon_{1}<1 .
\end{aligned}
$$

Therefore, for $\epsilon_{1}<1$ and

$$
\begin{aligned}
p & >\frac{\ln \left(1-\epsilon_{1}\right)}{\ln \left(\frac{1-\epsilon_{1}}{1+\epsilon_{0}}\right)} \\
& =\min \left\{\frac{\ln \left(1-\lambda_{1}\right)}{\ln \left(\frac{1-\lambda_{1}}{1-\lambda_{0}}\right)}:\left(\lambda_{0}, \lambda_{1}\right) \in\left(0, \frac{\epsilon_{0}}{1+\epsilon_{0}}\right] \times\left(-\infty,-\frac{\epsilon_{1}}{1-\epsilon_{1}}\right]\right\}
\end{aligned}
$$

we obtain

$$
\lim _{n \rightarrow \infty} 1_{\Omega \times[b, 1]}\left(T^{n}(\omega, x)\right)=0 \quad \text { for a.e. } \omega
$$

Similarly, for

$$
p<\frac{\ln \left(1+\epsilon_{1}\right)}{\ln \left(\frac{1+\epsilon_{1}}{1-\epsilon_{0}}\right)}=\max \left\{\frac{\ln \left(1-\lambda_{1}\right)}{\ln \left(\frac{1-\lambda_{1}}{1-\lambda_{0}}\right)}:\left(\lambda_{0}, \lambda_{1}\right) \in\left[\epsilon_{0}, 1\right] \times\left[-\epsilon_{1}, 0\right]\right\}
$$

we have

$$
\lim _{n \rightarrow \infty} 1_{\Omega \times[0, b]}\left(T^{n}(\omega, x)\right)=0 \quad \text { for a.e. } \omega .
$$

As a consequence we get

THEOREM 6. If $T$ is given by (3) then $\left(T, \mu_{p} \times \Lambda\right)$ is totally dissipative and the set of product measures in $M_{p}$ is $\operatorname{conv}\left\{\mu_{p} \times \delta_{\{0\}}, \mu_{p} \times \delta_{\{1\}}\right\}$ whenever

$$
p<\frac{\ln \left(1+\epsilon_{1}\right)}{\ln \left(\frac{1+\epsilon_{1}}{1-\epsilon_{0}}\right)} \quad \text { or } \quad p>\frac{\ln \left(1-\epsilon_{1}\right)}{\ln \left(\frac{1-\epsilon_{1}}{1+\epsilon_{0}}\right)} .
$$

Here $M_{p}$ denotes the set of $T$-invariant probability measures $m$ such that $m \mid \mathcal{B} \times\{[0,1]\}=\mu_{p}$

Proof. Let us assume the first inequality holds. Then

$$
\left\{(\omega, x): \sum_{n=1}^{\infty} 1_{\Omega \times[0, b]}\left(T^{n}(\omega, x)\right)<\infty\right\}
$$

has measure one. Therefore, by the Halmos recurrence theorem $[\mathrm{A}],[0, b] \subset$ $\mathcal{D}_{T}$ for every $0<b<1$. Hence $\mathcal{D}_{T}=[0,1]$. Moreover,

$$
\begin{aligned}
A^{n} I(x) & =\int_{0}^{x} P^{n} 1 d \Lambda=\int_{\Omega}^{1} \int_{0}^{n} P^{n} 1 \cdot 1_{\Omega \times[0, x]} d \Lambda d \mu_{p} \\
& =\int_{\Omega 0}^{1} \int_{\Omega \times[0, x]}\left(T^{n}(\omega, t)\right) d \Lambda d \mu_{p} \rightarrow 0 .
\end{aligned}
$$


Thus by Theorem 3 of [K3] we get the desired conclusion. The proof for the second inequality is similar.

4. The example. Let us consider the example from [K2]:

$$
T_{0}(x)=\frac{3}{2} x-\frac{1}{2} x^{2}, \quad T_{1}(x)=x^{2}, \quad \text { i.e. } \quad \epsilon_{0}=\frac{1}{2}, \quad \epsilon_{1}=1,
$$

and $T$ given as in (1).

Theorem 7. $M_{p}=\operatorname{conv}\left\{\mu_{p} \times \delta_{\{0\}}, \mu_{p} \times \delta_{\{1\}}, \mu_{p} \times \Lambda\right\}$ for $p=2 / 3$. The set of product measures in $M_{p}$ is $\operatorname{conv}\left\{\mu_{p} \times \delta_{\{0\}}, \mu_{p} \times \delta_{\{1\}}\right\}$ for $p \in(0,1 / 2]$. Moreover, $\left(T, \mu_{p} \times \Lambda\right)$ is totally dissipative for $p<1 / 2$.

REMARK 2 . We only need to prove the case $p=1 / 2$. The other conclusions result from Theorem 6 and Theorem 3 of [K3] respectively.

We will need the considerations below. Define the operator $\mathcal{A}$ on $\mathcal{D}$ as follows:

$$
\mathcal{A} F(x)=\frac{1}{2} F\left(T_{0}(x)\right)+\frac{1}{2} F\left(T_{1}(x)\right) \quad \text { for } F \in \mathcal{D} .
$$

Let $\nu_{F}$ denote the measure determined by $F$.

FACT ([K2]). The measure $\mu_{1 / 2} \times \nu_{F}$ is T-invariant if and only if $\mathcal{A} F$ $=F$.

LEMMA 1.

$$
\lim _{n \rightarrow \infty} \mathcal{A}^{n} I(x) \leq \frac{1}{2} \quad \text { for } x \in[0,1) .
$$

Proof. Since $T_{1}\left(T_{0}(x)\right) \leq I(x)$ for $x \in[0,1]$ we have

$$
\begin{aligned}
\mathcal{A}^{n} I(x) \leq & \frac{1}{2^{n}} \sum_{k=0}^{n}\left(\begin{array}{l}
n \\
k
\end{array}\right) x^{2^{2 k-n}} \\
\leq & \frac{1}{2^{n}} \sum_{k=0}^{E(n / 2)}\left(\begin{array}{l}
n \\
k
\end{array}\right) x^{2^{2 k-n}}+\frac{1}{2^{n}} \sum_{k=E(n / 2)}^{E(n / 2)+s}\left(\begin{array}{l}
n \\
k
\end{array}\right) \\
& +\left[\frac{1}{2^{n}} \sum_{k=E(n / 2)+s+1}^{n}\left(\begin{array}{l}
n \\
k
\end{array}\right)\right] x^{2^{s}} \\
\leq & \frac{1}{2}+\frac{1}{2^{n}} \sum_{k=E(n / 2)}^{E(n / 2)+s}\left(\begin{array}{l}
n \\
k
\end{array}\right)+\frac{1}{2} x^{2^{s}},
\end{aligned}
$$

where $E(x)$ denotes the integer part of $x$. By the existence of $\lim _{n \rightarrow \infty} \mathcal{A}^{n} I$ (see Lemma 3 of [K2]) we get

$$
\lim _{n \rightarrow \infty} \mathcal{A}^{n} I(x) \leq \frac{1}{2}+\frac{1}{2} x^{2^{s}} \quad \text { for any } s \geq 1 .
$$

Therefore $\lim _{n \rightarrow \infty} \mathcal{A}^{n} I(x) \leq 1 / 2$ for $x \in[0,1)$. 
Lemma 2. Let $\omega(x)$ be a polynomial such that $0 \leq \omega(x) \leq \delta$ for $x \in$ $[0,1], \omega(0)=0$ and $\delta<1$. Then $\lim \sup _{n \rightarrow \infty} \mathcal{A}^{n} \omega(x) \leq 1 / 2$ for $x \in[0,1)$.

Proof. We first observe that

$$
\limsup _{n \rightarrow \infty} \mathcal{A}^{n} I^{1 / k}(x) \leq \frac{1}{2} \quad \text { for } x \in[0,1) \text { and } k=1,2, \ldots
$$

Let

$$
d_{n}(x)=\frac{1}{2^{n}} \sum_{k=0}^{n}\left(\begin{array}{l}
n \\
k
\end{array}\right) x^{2^{2 k-n}} .
$$

By the proof of Lemma 1 we see that $\mathcal{A}^{n} I^{1 / k}(x) \leq d_{n}\left(x^{1 / k}\right)$ and naturally

$$
\limsup _{n \rightarrow \infty} \mathcal{A}^{n} I^{1 / k}(x) \leq \frac{1}{2}+\frac{1}{2} x^{2^{s} / k} \quad \text { for } s=1,2, \ldots
$$

Therefore $\lim \sup _{n \rightarrow \infty} \mathcal{A}^{n} I^{1 / k} \leq 1 / 2$ for $x \in[0,1)$. Let $\omega(x)$ be a polynomial satisfying our assumptions. Then $\omega(x) \leq x^{1 / 2}$ for $x \in[0, \epsilon]$ and for some $\epsilon>0$. If we take $k$ such that $\epsilon^{1 / k}>\delta$ then $\omega(x) \leq x^{1 / k}$ for $x \in[0,1]$. Hence $\mathcal{A}^{n} \omega \leq \mathcal{A}^{n} I^{1 / k}$ and as a result

$$
\limsup _{n \rightarrow \infty} \mathcal{A}^{n} \omega(x) \leq \limsup _{n \rightarrow \infty} \mathcal{A} I^{1 / k}(x) \leq \frac{1}{2} \quad \text { for } x \in[0,1) .
$$

Lemma 3. Let $T$ be given by (2) and let $\mu_{p} \times \nu_{p}$ be a T-invariant measure. If $\nu_{p} \notin \operatorname{conv}\left\{\delta_{\{0\}}, \delta_{\{1\}}\right\}$ then $\nu_{F}$ has the dense support property (equivalently $F$ is 1-1).

Proof. Let $T_{0}=\left(1+\epsilon_{0}\right) x-\epsilon_{0} g(x)$ and $T_{1}(x)=\left(1-\epsilon_{1}\right) x+\epsilon_{1} g(x)$ for $\epsilon_{0}, \epsilon_{1}>0$. Then

$$
T_{0}(x)=(1+\epsilon) x-\epsilon T_{1}(x) \quad \text { for } \epsilon=\epsilon_{0} / \epsilon_{1} .
$$

Let $(a, b)$ be a nonempty interval of maximal length such that $F \mid(a, b)=$ const. By assumptions we have $(a, b) \neq(0,1)$ and $T_{0}(b)-T_{0}(a) \leq b-a$, $T_{1}(b)-T_{1}(a) \leq b-a$. Here we use the fact that $F \mid\left(T_{0}(a), T_{0}(b)\right)=$ const and $F \mid\left(T_{1}(a), T_{1}(b)\right)=$ const by Lemma 1 of [K2]. In particular,

$$
\begin{aligned}
T_{0}(b)-T_{0}(a) \leq b-a & \Leftrightarrow(1+\epsilon) b-\epsilon T_{1}(b)-(1+\epsilon) a+\epsilon T_{1}(a) \leq b-a \\
& \Leftrightarrow T_{1}(b)-T_{1}(a) \geq b-a .
\end{aligned}
$$

Therefore, $T_{1}(b)-T_{1}(a)=b-a$ and by induction $T_{1}^{n}(b)-T_{1}^{n}(a)=b-a$ for $n=1,2, \ldots$. Hence $a=b$.

Proof of Theorem 7. Suppose, contrary to our claim, that there exists a product measure in $M_{1 / 2}$ outside $\operatorname{conv}\left\{\mu_{p} \times \delta_{\{0\}}, \mu_{p} \times \delta_{\{1\}}\right\}$. We may assume (by ergodic decomposition [Ki]) that there exists a distribution $G$ such that $\mu_{p} \times \nu_{G}$ is ergodic and $\nu_{G} \notin \operatorname{conv}\left\{\delta_{\{0\}}, \delta_{\{1\}}\right\}$. Since $\nu_{G}$ has the dense support property (by Lemma 3 ) we see that $G$ is continuous and increasing. Therefore 
for every $\epsilon>0$ there exists a polynomial $\omega_{\epsilon}$ such that $\omega_{\epsilon}(0)=0, \quad 0 \leq \omega_{\epsilon}(x) \leq 1-\epsilon \quad$ for $\quad x \in[0,1] \quad$ and $\quad\left\|G-\omega_{\epsilon}\right\| \leq 3 \epsilon$.

Thus we obtain

$$
\left\|\mathcal{A}^{n} G-\mathcal{A}^{n} \omega_{\epsilon}\right\|=\left\|G-\mathcal{A}^{n} \omega_{\epsilon}\right\| \leq 3 \epsilon
$$

and $G \leq 1 / 2$ for $x \in[0,1)$ by Lemma 2 . This contradicts our assumption.

Acknowledgments. Research supported by grant MENII 1 P03A 021 29, Poland.

\section{References}

[A] J. Aaronson, An Introduction to Infinite Ergodic Theory, Math. Surveys Monogr. 50, Amer. Math. Soc., 1997, p. 284.

[D-K-S] M. Denker, Y. Kifer and M. Stadlbauer, Conservativity of random Markov fibred systems, Ergodic Theory Dynam. Systems 28 (2008), 67-86.

[D-L] Y. Derriennic et M. Lin, Sur la tribu asymptotique des marches aléatoires sur les groupes, Publ. Sém. Math., Univ. Rennes I, Rennes, 1983.

[Ki] Y. Kifer, Ergodic Theory of Random Transformations, Progr. Probab. Statist. 10, Birkhäuser, 1986, p. 210.

[K1] Z. S. Kowalski, Stationary perturbations based on Bernoulli processes, Studia Math. 97 (1990), 53-57.

[K2] -, Invariant measures for smooth extensions of Bernoulli shifts, Bull. Polish Acad. Sci. 51 (2003), 261-267.

[K3] —, Smooth extensions of Bernoulli shifts, ibid. 53 (2005), 157-168.

Institute of Mathematics and Computer Science

Wrocław University of Technology

Wybrzeże St. Wyspiańskiego 27

50-370 Wrocław, Poland

E-mail: kowalski@pwr.wroc.pl

Received 4 September 2007;

in revised form 10 December 2008 